\title{
Inbreeding and extreme outbreeding cause sex differences in immune defence and life history traits in Epirrita autumnata
}

\author{
MJ Rantala ${ }^{1}$ and DA Roff ${ }^{2}$ \\ ${ }^{1}$ Department of Biology, Section of Ecology, University of Turku, Turku, Finland and ${ }^{2}$ Department of Biology, University of California, \\ Riverside, CA, USA
}

\begin{abstract}
Empirical studies in vertebrates support the hypothesis that inbreeding reduces resistance against parasites and pathogens. However, studies in insects have not found any evidence that inbreeding compromises immune defence. Here we tested whether one generation of brother-sister mating or extreme outbreeding (mating between two populations) have an effect on innate immunity and life history traits in the autumnal moth, Epirrita autumnata. We show that the effect of inbreeding on immune response differed between the sexes: whereas in females, inbreeding significantly reduced encapsulation response against nylon monofilament ability, it did not have a significant effect on male immune response. There were also differences in the correlation of the immune response with other traits: in females increased immune response was positively correlated with large size, whereas in males immune response
\end{abstract}

increased with a reduction in development time. Immune response differed significantly among families in males but not in females, both for the inbreeding and extreme outbreeding experiments. In conjunction with the observed immune responses to inbreeding, these data suggest that in males genetic variation for immune response is largely additive or non-directional with respect to dominance, whereas in females variation is much reduced and consists of directional dominance variance. Further, we show that encapsulation response against nylon monofilament is associated with the resistance against real pathogens suggesting that this widely used method to measure the strength of immune defence in insects is also a biologically relevant method.

Heredity (2007) 98, 329-336. doi:10.1038/sj.hdy.6800945; published online 21 February 2007

Keywords: Beauveira bassiana; encapsulation; immune function; immunocompetence; pathogens; sexual dimorphism

\section{Introduction}

The loss of habitat and habitat fragmentation has become a serious problem for many species (reviewed in Hanski, 2005). Habitat fragmentation leads to increased isolation and decreased population size that may lead to the erosion of genetic variation and increased genetic differentiation among populations through genetic drift, increased inbreeding and reduced gene flow between populations. These effects together or singly may have serious consequences for the persistence of fragmented populations (Hanski, 2005). Among vertebrates there is an abundance of evidences that inbreeding, the mating of close relatives, can increase their susceptibility to pathogens by reducing their immune defence (reviewed in Keller and Waller, 2002). However, these studies contrast with studies on invertebrates in which no such depression has been observed. For example, in the bumble-bee, Bombus terrestris (L.), inbreeding (brothersister mating) did not have any effect on innate immune defence or body size of the offspring (Gerloff et al., 2003). In the termite, Zootermopisis angusticollis, inbreeding

Correspondence: Dr MJ Rantala, Department of Biology, Section of Ecology, University of Turku, FIN-20014 Turku, Finland.

E-mail:marrant@cc.jyu.fi

Received 21 October 2006; revised 10 January 2007; accepted 15 January 2007; published online 21 February 2007 neither decreased encapsulation response nor resistance against bacterial or fungal disease (Calleri et al., 2006). Similarly, inbred lines of the flour beetle Tribolium castaneum did not differ from a stock population in their resistance to parasitic nematodes (Stevens et al., 1997). Finally, in the sand cricket, Gryllus firmus, individuals from inbred lines were smaller and took longer to develop than individuals from crosses between lines but did not differ in their immune function (Rantala and Roff, 2006). Given the differences between results for vertebrates and invertebrates, more studies on insects are needed in this field, especially on species that do not normally experience inbreeding, because ancestral inbreeding may reduce the magnitude of inbreeding depression (see Swindell and Bouzat, 2006). Additionally, there are no data bearing on the opposite question, namely whether crosses between populations exhibit heterosis or outbreeding depression for immune response.

Insects are excellent models for studying the effect of inbreeding and heterosis on immune defence, because the immune defence system in insects is far less complex than the vertebrate immune system, even though many components are homologous (Vilmos and Kurucz, 1998). Further, because they do not possess lymphocytes or immunoglobulins, most insects do not show an acquired immunity (Gillespie et al., 1997). Insect immunity is characterized by the inducible expression of a large array 
of antimicrobial peptides and by the constitutive melanization-encapsulation response. Encapsulation is an immune response through which insects defend themselves against endoparasitoid wasps and flies (Salt, 1970), fungi, nematodes and bacteria (reviewed in Gupta 2001). Encapsulation is an immune response in which hemocytes recognize an object as foreign and cause other hemocytes to aggregate and form a capsule and a cascade of biochemical reactions lead to the deposition of melanin and the hardening of the capsule (Gillespie et al., 1997). The enclosed intruder dies from suffocation or from the release of necrotizing compounds (Nappi et al., 1995). In invertebrates, one of the most informative ways to assay this reaction is to measure the magnitude of the encapsulation response to a novel and standardized antigen such as a nylon monofilament (e.g. Rantala et al., 2000, 2003a, b; Fedorka et al., 2004; Koskimäki et al., 2004; Zuk et al., 2004; Simmons et al., 2005). It has been shown that the ability to encapsulate abiotic material is strongly related to the ability to encapsulate a parasite (Paskewitz and Riehle, 1994; Gorman et al., 1996). However, to our knowledge, there are no studies testing whether encapsulation response against a nylon monofilament is associated with resistance against real pathogens. Given the frequent use of this technique as an index of immune response such testing is strongly warranted.

The purpose of the present study was to answer three questions using the autumnal moth, Epirrita autumnata, as the model organism: (1) is the encapsulation response of nylon monofilament associated with the ability to resist real pathogens; (2) does inbreeding or heterosis have an effect on innate immunity response or other traits that may be correlated with this response, specifically development time, pupal mass and survival; (3) are such effects sex-specific? To address the second two questions we first compared full sib families from unrelated parents to those in which sires and dams were full sibs (i.e. brother-sister mating) and secondly we compared offspring from crosses between two populations to crosses within each of the two original populations.

\section{Materials and methods}

\section{Study organism}

The autumnal moth, E. autumnata, is a holarctic geometrid with a univoltine life cycle. The larvae eclose in spring in synchrony with birch leaf flush and the larvae feed on the young foliage, the chemical and physical quality of which decreases rapidly during the larval period (reviewed in Haukioja, 2003). Both sexes have five larval stages and the larval period lasts for about 1 month. Pupation occurs in the ground during midsummer, and the adults eclose in autumn. The shortlived nocturnal adults have a weakly developed proboscis and do not feed but support maintenance and reproduction on stored reserves (e.g. Tammaru et al., 1996). Thus, their reproductive capacity is directly dependent on the body mass accumulated during the larval stage and can be reliably indexed by pupal mass (Tammaru et al., 1996). The autumnal moth is famous for massive outbreaks that cause extensive damage to entire mountain birch forests in the northern and the mountainous part of its distribution. These outbreaks occur with a periodicity of 9-10 years and last for 2-3 years (Haukioja, 2005). In northern Finland, more than 10 parasitoid species are known to parasitize E. autumnata (Ruohomäki et al., 2000). Further, in E. autumnata, entomopathogenic fungus, bacteria and viruses impose significant morbidity and mortality costs on natural populations (Rantala et al., unpublished). Thus, there should be strong selection for an effective immune defence in field populations. However, the immune defence of the autumnal moth has varied with genotype (van Ooik et al., 2007), food quality (Kapari et al., 2006), food quantity (Yang et al., 2007), larval density (Kilpimaa et al., unpublished) and with the level of heavy metal pollution (van Ooik et al., 2007). Given the observed variation with genotype, the effects of outbreeding and inbreeding on the immune system are pertinent questions.

\section{Effect of inbreeding on innate immune defence and other life history traits}

The grandparents of the experimental larvae were collected as larvae (about 80 ) from the area around the Kevo Subarctic Research Station of Turku University $\left(69^{\circ} 45^{\prime} \mathrm{N}, 27^{\circ} 01^{\prime} \mathrm{E}\right) 2$ years before the start of these experiments. During the collection of larvae, we minimized the probability that such larvae were related to each other by collecting each larva from different trees. Parents of the experimental larvae originated from laboratory matings of these individuals. During the next summer, parents were reared in full sib groups in buckets (101) and fed with fresh leaves. These parents were mated to create 15 inbred $(\mathrm{F}=0.25$, parents brother-sister) and 15 outbred families.

From each family we randomly chose 20 newly hatched larvae giving an initial sample of 600 larvae. The larvae were reared individually in $48 \mathrm{ml}$ vials in outdoor conditions and provided with fresh mountain birch leaves ad libitum during their entire larval period. After the fifth larval instar and short prepupal stage, larvae were allowed to pupate individually into moist Sphagnum moss. The length of larval period was determined as days between hatching and the beginning of the prepupal period. Before the immune assays (see below) the pupae were kept in a laboratory at $+14^{\circ} \mathrm{C}$ for 7 days. Prior to the immune assays, pupal mass was measured to the nearest $0.1 \mathrm{mg}$ and sex determined.

\section{Effect of outbreeding on innate immune defence and other life history traits}

To test the effect of heterosis on the immune system, two separate populations of E. autumnata larvae from two localities, Kevo (Northern Finland) and Abisko (Northern Sweden), were used. The Abisko population is noted for its extreme fluctuations and achieving outbreak densities, whereas, because of climatic factors, the Kevo population fails to reach outbreak densities but shows similar cyclical fluctuations in density. The distance between these two localities is about $370 \mathrm{~km}$, but each locality is surrounded by mountains and fells and thus is geographically isolated. Although geographically widely separated, some gene flow is potentially possible, as the species has a transholarctic distribution. The grandparents of the experimental larvae were collected as larvae (about 100) from the area around the Abisko 2 
years before the start of these experiments. As in the case of the Kevo population, we minimized the probability of larvae being related by collecting each larva from a different tree. Parents of the experimental larvae originated from the laboratory matings of these individuals. During the next summer, parents were reared up in full sib groups in buckets (101) and fed with fresh leaves.

Parents were mated to create 15 'within-Kevo' populations families (outbred, same as used as control families in the inbreeding experiment), 15 'within-Abisko' families (outbred) and 15 hybrid families (seven sires from Kevo and eight sires from Abisko). From each family we randomly chose 20 newly hatched larvae to give an initial sample of 300 larvae per locality/ treatment (900 larvae in total). Rearing and treatment before the immune assay were the same as described for the inbreeding experiment.

\section{Encapsulation rate assay}

To measure encapsulation rate, we inserted a $2 \pm 0.1 \mathrm{~mm}$ length of nylon monofilament (diameter $0.18 \mathrm{~mm}$, rubbed with sandpaper and knotted) into the abdomen of each pupa. This treatment enhanced the likelihood that hemocytes would stick to the implant and facilitated removal of the implant. After insertion of the monofilament, the immune system of a pupa was allowed to react to this 'implant' for $1 \mathrm{~h}$ at $22^{\circ} \mathrm{C}$. Our previous studies in this species indicated that $1 \mathrm{~h}$ gave the highest variance between individuals in encapsulation rate, whereas still not at an equilibrium (Kapari et al., 2006; van Ooik et al., 2007). After $1 \mathrm{~h}$, pupae were frozen and stored at $-80^{\circ} \mathrm{C}$. The implant was later removed from each pupa and the monofilament photographed from two different angles under a light microscope with a digital camera recorder. These pictures were analysed using the Image Pro program. The degree of encapsulation was analysed as grey values of reflecting light from implants. As a measure of encapsulation rate, we used the average grey values of two digital pictures. The data were transformed so that the darkest grey values corresponded to the highest encapsulation rate. This transformation was carried out by subtracting the observed grey values from the control grey value (clear implant) (see Rantala and Kortet, 2003; Rantala et al., 2004). In previous studies, the repeatability of the method to measure the darkness of the implant has been found to be very high, ranging from 0.82 to 0.997 (Rantala et al., 2002; Rantala and Roff, 2006).

\section{Encapsulation response and the resistance against real pathogens}

The larvae used in this experiment were collected as last (fifth) instar larvae from the outbreak area from the fell Nuvvus Ailigas. Before the experiment, the mass of larvae was measured to the nearest 0.1. Unfortunately, we were not able to determinate the sex of larvae. To measure encapsulation response against nylon monofilament, we inserted a $2 \pm 0.1 \mathrm{~mm}$ length of nylon monofilament (diameter $0.18 \mathrm{~mm}$, rubbed with sandpaper and knotted) into the fourth segment of a larva. We allowed the larva to react to this 'implant' for $1 \mathrm{~h}$ at $22^{\circ} \mathrm{C}$ after which we smoothly removed the implant for later analysis. During the next day, the surviving larvae $(N=62)$ were exposed to the fungus, Beauveria bassiana (strain Mycotrol, suspended in inert ingredients and distilled water), by dipping them in $10 \mathrm{ml}$ of an $\mathrm{LD}_{50}$ dose solution for $5 \mathrm{~s}$. We determined $\mathrm{LD}_{50}$ doses by giving a separate group of larvae five increasing doses of conodians and selecting the dose that will came closest to killing $50 \%$ of the animals $\left(3.55 \times 10^{6}\right.$ spores $\left./ \mathrm{ml}\right)$. The larvae were individually incubated in $48 \mathrm{ml}$ vials at $22^{\circ} \mathrm{C}$ and fed with fresh leaves until pupation. The survival of larvae was checked daily until pupation. Larvae that pupated successfully were recorded as survived. The inert ingredients used with conidians did not cause any mortality in preliminary experiments. Therefore, we dipped the controls in distilled water without fungal spores or inert ingredients. There was no natural mortality among the control group of 60 individuals and they were therefore excluded from the statistical analysis. Unfortunately, the mortality because of fungus was much higher in the experiment than in preliminary studies (probably because of the injury by the nylon monofilament) and mortality was $68 \%$.

\section{Statistical analysis}

Excluding survival, for which sex could not be assigned, all traits were distinguished by sex, family membership, inbreeding coefficient $(\mathrm{F}=0$ or 0.25$)$ and parental category (for crosses between and within populations). Genetic variability in traits within populations is indicated by variation among families from crosses within populations and response to inbreeding. Crosses between populations can be used to assess genetic variation between populations but not within populations. In principle, the effect of sex and inbreeding status could be assessed by nesting family within these components. However, such a model potentially includes a large number of interaction terms, which could reduce the power of finding main effects. To avoid these problems we proceeded in two steps: first, we ignored the potential effects of family membership and tested for variation because of sex and breeding status (inbred or outbred) or cross type (two populations and hybrid) using two-way ANOVAs. These preliminary analyses (see below) indicated that both sex, inbreeding status or cross type could be significant and so we did separate analyses for each sex, using a nested ANOVA, with family nested within inbreeding status or cross type. Because it is likely that traits are correlated, we next proceeded in the case of the inbred experiment with a stepwise multiple regression analysis using either the entire data set or the mean family values for each sex. In the latter case the resulting regressions are indicative of genetic correlations among parameters.

\section{Results}

\section{Encapsulation response and the resistance against real pathogens}

Larvae that survived the Beauviria bassiana infection had higher encapsulation response before infection than those that died ( $t$-test, $t=-2.056, \mathrm{df}=60, P=0.044$ ). There was also no differences in body mass before infection between those who survived and those who died ( $t$-test, $t=-1.121, \mathrm{df}=60, P=0.267)$. We conclude that the encapsulation assay is a suitable index of the resistance to $B$. bassiana infection. Whether it is also 
positively correlated to the response to other pathogens remains to be tested.

Effect of inbreeding on innate immune defence and other life history traits

Analysis of the data where family membership is ignored (Figure 1) shows that (1) outbred males and females were very similar with respect to encapsulation value but inbred individuals showed a lowered response, with females showing the greatest decline; (2) development time of females was significantly longer than males and outbred individuals developed in significantly faster than inbred individuals, with females again showing the greatest response to inbreeding, but the interaction between sex and status was not significant; (3) males were significantly smaller than females and outbred individuals were heavier than inbred individuals, though the difference was marginally nonsignificant. Additionally, the survival rate of outbred larvae was higher than the survival rate of the inbred larvae (72.11 and 50.67\%, respectively).

Results for the nested ANOVAs testing for family (genetic) and inbreeding effects within the two sexes are summarized in Table 1. Encapsulation rate in males was highly significantly variable among families but not associated with inbreeding status. In contrast, in females there was no significant variation among families but a highly significant difference with breeding status, inbred females showing a reduced encapsulation rate (Figure 1).

Significant variation among families was found in all other traits with the exception of development time in females. Highly significant differences because of inbreeding status were found in development time of females and survival in both sexes, but effects were only marginally significant for development time in males and pupal mass in females, and not significant for mass in males (Table 1).

To assess the joint effect of inbreeding status, development time and pupal mass on encapsulation rate, we
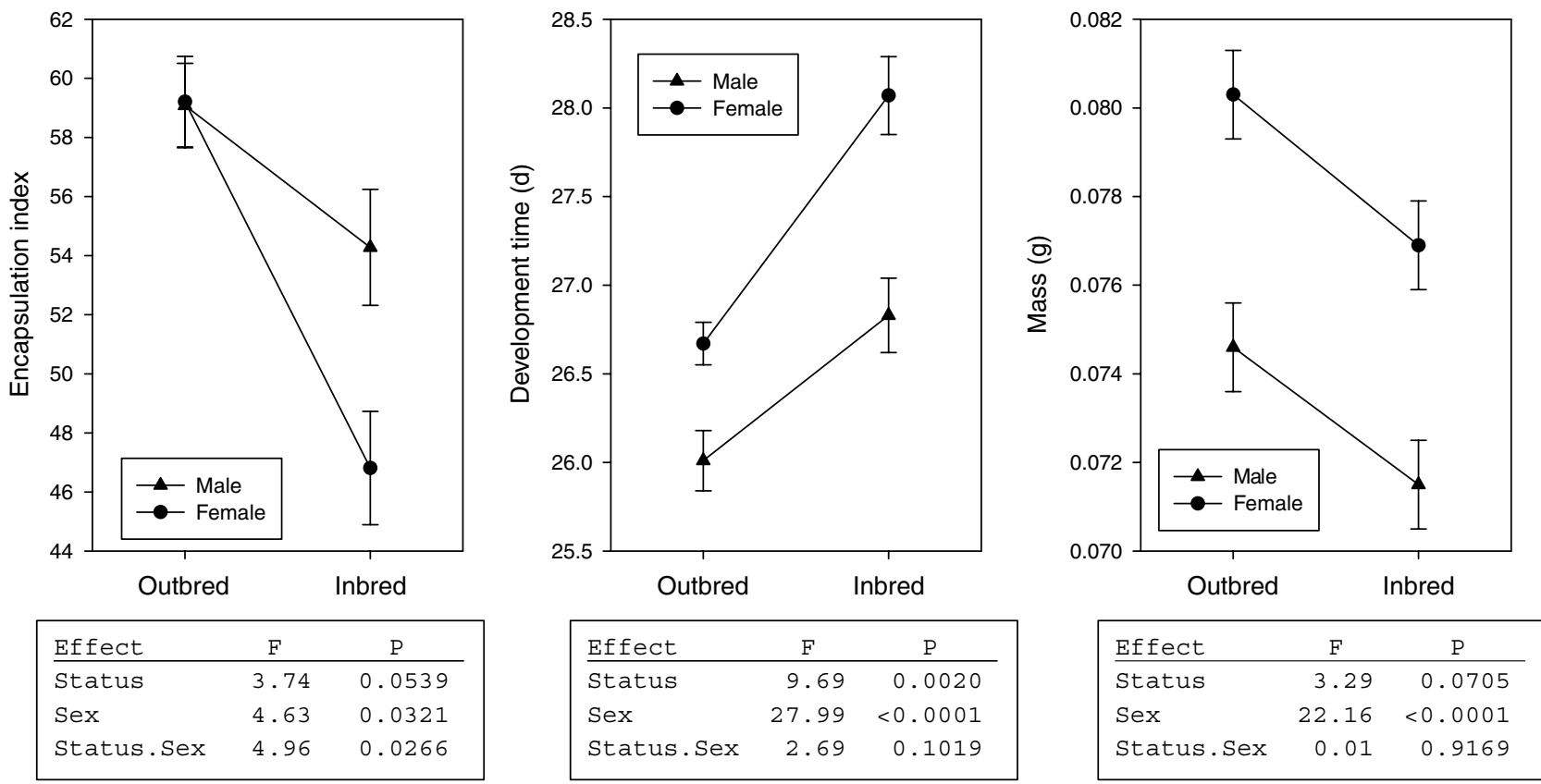

Figure 1 Effects of sex and breeding status on encapsulation index, development time and pupal mass in the wax moth. Sample sizes: males = 102 (outbred), 69 (inbred); females $=108$ (outbred), 82 (inbred).

Table 1 Nested ANOVAs testing for genetic variation among families (Family) and with inbreeding status (Status)

\begin{tabular}{|c|c|c|c|c|c|c|c|c|c|}
\hline \multirow[t]{2}{*}{ Trait } & \multirow[t]{2}{*}{$\operatorname{Sex}$} & \multicolumn{4}{|c|}{ Family } & \multicolumn{4}{|c|}{ Status } \\
\hline & & $F$ & $d f 1$ & $d f 2$ & $\mathrm{P}$ & $F$ & $d f 1$ & $d f 2$ & $\mathrm{P}$ \\
\hline Encapsulation & Male & 2.03 & 27 & 142 & 0.0041 & 2.26 & 1 & 27 & 0.1444 \\
\hline Encapsulation & Female & 0.80 & 28 & 160 & 0.7462 & 10.06 & 1 & 28 & 0.0037 \\
\hline Development time & Male & 2.03 & 27 & 142 & 0.0042 & 5.04 & 1 & 27 & 0.0332 \\
\hline Development time & Female & 1.25 & 28 & 160 & 0.1959 & 28.21 & 1 & 28 & $<0.0001$ \\
\hline Pupal mass & Male & 2.04 & 27 & 142 & 0.0040 & 0.90 & 1 & 27 & 0.3512 \\
\hline Pupal mass & Female & 2.88 & 28 & 160 & $<0.0001$ & 5.37 & 1 & 28 & 0.0281 \\
\hline Survival $^{\mathrm{a}}$ & Both & 4.66 & 28 & 562 & $<0.0001$ & 7.30 & 1 & 28 & 0.0116 \\
\hline
\end{tabular}

Abbreviation: ANOVA, analysis of variance.

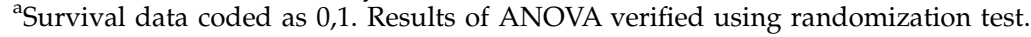


employed a stepwise multiple regression for each sex separately. For each sex the final model was statistically significant (females, $\mathrm{F}_{3,186}=12.78, P<0.0001$; males, $\left.\mathrm{F}_{2,168}=5.05, P=0.0074\right)$, but whereas that for females consisted of breeding status and mass, that for males consisted of breeding status and development time. Encapsulation rate of females increased with pupal mass but was reduced among inbred females with pupal weight. In males, encapsulation rate was decreased with both inbreeding and development time. The final model for females accounted for $17.09 \%$ of the variance but that for males accounted for only $5.67 \%$. Using family mean values resulted in a significant final model for females $\left(\mathrm{F}_{1,28}=34.91, P<0.0001\right)$ that contained only breeding status and accounted for $55.49 \%$ of the variance. Both breeding status and development time were retained in the final model for males, which accounted for a similar percentage $(46.58 \%)$ of the variance as in females. Considering these regressions overall, they indicate that high immune response in individuals (or genotypes) is associated with breeding status, short development time and large adult mass. In males, there is evidence for a genetic correlation with development time but not in females, though the statistical power of the tests is low, given the small number of families.

\section{Effect of outbreeding on innate immune defence and other life history traits}

Two-way analysis of variance, ignoring family membership (Figure 2), shows that (1) encapsulation values vary significantly only with the type of cross, with the hybrids showing a higher encapsulation response; (2) development time of females was significantly longer than males and there was significant variation among the crosses, with hybrids developing the fastest; (3) males were significantly smaller than females and there was significant variation among the crosses, with hybrids the heaviest individuals.

Results for the nested ANOVAs testing for genetic (family) and among-cross effects within the two sexes are summarized in Table 2. As with the inbreeding results, encapsulation rate in males was highly significantly variable among families but variation among families was not significant in females. Males showed no significant variation among crosses whereas significant

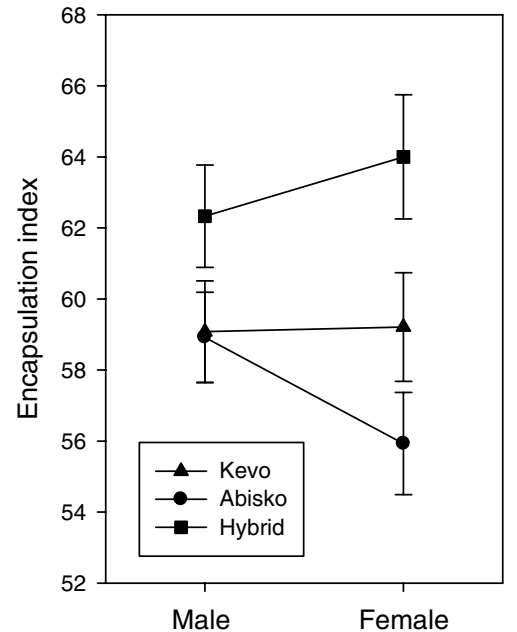

\begin{tabular}{|lcc|}
\hline Effect & F & P \\
\hline Cross & 7.92 & 0.0004 \\
Sex & 0.11 & 0.7425 \\
Status.Sex & 1.30 & 0.2740 \\
\hline
\end{tabular}

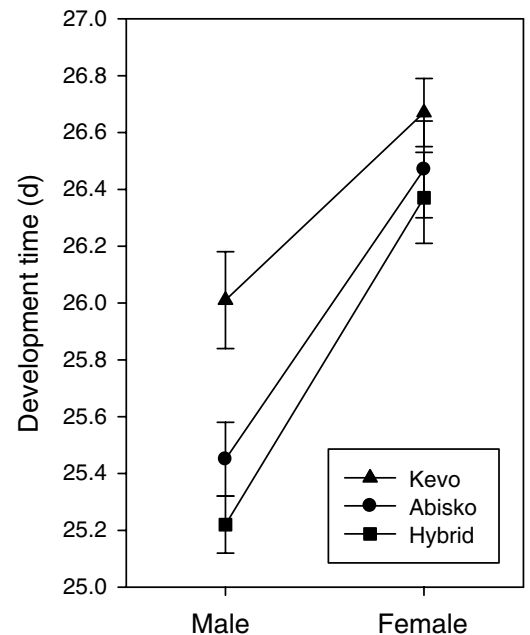

\begin{tabular}{|lrr|}
\hline Effect & \multicolumn{1}{c}{ F } & \multicolumn{1}{c|}{ P } \\
\hline Cross & 7.17 & 0.0008 \\
Sex & 62.73 & $<0.0001$ \\
Cross.Sex & 1.50 & 0.2247
\end{tabular}

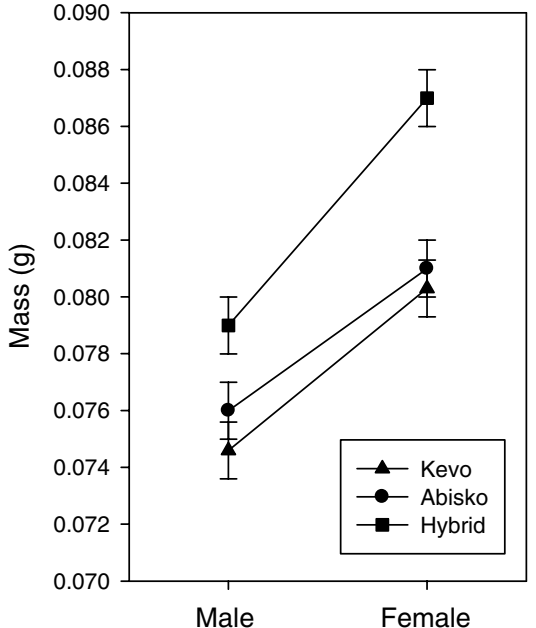

\begin{tabular}{|lrr|}
\hline Effect & \multicolumn{1}{c}{$\mathrm{F}$} & \multicolumn{1}{c|}{$\mathrm{P}$} \\
\hline Cross & 17.02 & $<0.0001$ \\
Sex & 62.70 & $<0.0001$ \\
Cross.Sex & 1.17 & 0.3110
\end{tabular}

Figure 2 Effects of sex and cross type on encapsulation index, development time and pupal mass in the wax moth. Sample sizes: Kevo males =102, Kevo females =108, Abisko males=110, Abisko females =110, Hybrid males =120, Hybrid females =92.

Table 2 Nested ANOVAs testing for genetic variation among families (Family) and with type of cross (Cross)

\begin{tabular}{|c|c|c|c|c|c|c|c|c|c|}
\hline \multirow[t]{2}{*}{ Trait } & \multirow[t]{2}{*}{ Sex } & \multicolumn{4}{|c|}{ Family } & \multicolumn{4}{|c|}{ Cross } \\
\hline & & $F$ & $d f 1$ & $d f 2$ & $\mathrm{P}$ & $F$ & $d f 1$ & $d f 2$ & $\mathrm{P}$ \\
\hline Encapsulation & Male & 1.58 & 42 & 285 & 0.0173 & 0.41 & 2 & 42 & 0.6687 \\
\hline Encapsulation & Female & 1.15 & 42 & 264 & 0.2486 & 5.96 & 2 & 42 & 0.0053 \\
\hline Development time & Male & 1.43 & 42 & 287 & 0.0503 & 6.49 & 2 & 42 & 0.0035 \\
\hline Development time & Female & 1.77 & 42 & 265 & 0.0040 & 0.12 & 2 & 42 & 0.8877 \\
\hline Pupal mass & Male & 1.97 & 42 & 287 & 0.0007 & 4.37 & 2 & 42 & 0.0188 \\
\hline Pupal mass & Female & 1.65 & 42 & 265 & 0.0100 & 4.11 & 2 & 42 & 0.0235 \\
\hline
\end{tabular}

Abbreviation: ANOVA, analysis of variance. 
variation was found in females. Significant variation among families and crosses was found in all other traits with the exception of development time in females that did not vary among crosses (Table 2 ).

\section{Discussion}

The encapsulation response against a piece of nylon monofilament is widely used and a standard method to measure for analyzing arthropods immunity (e.g. Vainio et al., 2004; Zuk et al., 2004; Ahtiainen et al., 2004, 2005; Rantala and Roff, 2005; Simmons et al., 2005), although there has been no direct evidence that it would reflect resistance against real pathogens and parasites. Mallon et al. (2003) found in B. terrestris that bumblebee colonies that were more resistant against gut trypanosomal parasites had a lower encapsulation response against the nylon monofilament. These results suggest a trade-off between specific and nonspecific components of the immune system at the colony level (Mallon et al., 2003). Unfortunately, they did not measure the association between encapsulation response and resistance against parasites at an individual level. In this study, we found that larvae that had a stronger encapsulation response against nylon monofilament had higher survival against $B$. bassiana than larvae with a weaker encapsulation against nylon monofilament. To our knowledge, this is the first study to show that encapsulation response against nylon monofilament is associated with the resistance against real pathogens at an individual level, which suggests that the method is biologically relevant.

We found that in E. autumnata there was strong inbreeding depression in all traits measured: inbreeding depressed survival, increased development times and decreased body mass. In contrast to previous studies in insects, which found no evidence that inbreeding would compromise the immune system (e.g. Stevens et al., 1997; Gerloff et al., 2003; Calleri et al., 2006), we found that inbreeding reduced a female's ability to encapsulate nylon monofilament markedly. However, this effect was not found in males after taking into account variation within families (Table 2; though a reduction considerably less than observed in inbred females was observed if family effects were ignored; Figure 1). These results indicate that the immune system of females is more vulnerable to inbreeding depression than the immune system of males in E autumnata. A possible cause of the difference between our current study and previous studies (e.g. Stevens et al., 1997; Gerloff et al., 2003; Calleri et al., 2006) is that in the previous studies the species examined may typically experience inbreeding in nature (e.g. in the latter two cited studies both species were social insects, termites and bees), which could have selected for a reduced effect of inbreeding on the immune system. The autumnal moth is a very common and abundant species of the northern hemisphere and capable of wide dispersal. Thus, it is very likely that mating between relatives is rare and the species has not recently passed through any genetic bottlenecks.

If the sex differences in vulnerability of immune defence to inbreeding depression observed in this study exists also among other taxa, it could have important consequence to persistence of small isolated inbred populations, especially if the observed sex differences in immune defence lead to sex differences in mortality to parasites and pathogens. This might also be a contributing factor to the male biased sex ratio that is often found in small populations before the extinction of that population (see Gabriel and Ferriere, 2004). It is known that females are more sensitive to environmental stress than males (Teder and Tammaru, 2005): the results of the present study suggest that part of this sensitivity is genetically based.

In vertebrates, males typically suffer more than females from parasitic infections and also tend to have reduced immune responses relative to females (reviewed in Keller and Waller, 2002), which has been suggested to be a consequence of the detrimental side effects of testosterone on male immunity (reviewed in Zuk and Stoehr, 2002). It has been suggested that the ultimate mechanism for sex differences in immune function would be a differential selection on the sexes favouring different investment levels in immune defence (see Zuk and Stoehr, 2002). Because male fitness is limited by the number females fertilized, whereas female fitness is limited by the number of offspring produced (Trivers, 1972), males are expected to invest more in sexual competition and current reproduction at the expense of their immune defence than females (see Zuk and Stoehr, 2002). Thus, the disparity in how sexes allocate resources is hypothesized to result in sexual dimorphism in immune defence even among taxa lacking testosterone (see Zuk and Stoehr, 2002).

However, although there are many studies in insects (e.g. Gray, 1998; Kurtz et al., 2000; Adamo et al., 2001; Vainio et al., 2004; Schwarzenbach et al., 2005) that have found a dimorphic immune response, there are also many studies in invertebrates that have failed to give support to this hypothesis. For example, Sheridan et al. (2000) found no sex differences in parasite infections among arthropod hosts. Likewise, efforts to examine the sex differences in immune function in insects have met with mixed results (see e.g. Rantala et al., 2007 and references therein). Recently, McKean and Nunney (2005) found that sex-specific responses to experimental manipulation of fitness-limiting resources affects both the magnitude and direction of sex differences in immune function in Drosophila melanogaster. They suggested that for species similarly limited in their reproduction, phenotypic plasticity would be an important determinant of sex differences in immune function and other life-history traits. In this study we found that immunological sex differences may vary in populations differing in their degree of inbreeding. Thus, it seems that there are plausible explanations for sexual dimorphism in immunity other than just the Bateman principle, which is traditionally used to explain the observed sex difference in immunity (for a review see Zuk and Stoehr, 2002).

Importantly, we found that males and females differed not only in the response of their immune systems to inbreeding but also in the extent to which their immune response was genetically variable, as measured by variation among families (Tables 1 and 2). In males the presence of genetic variation among families but no significant response to inbreeding indicates that most of the variation is additive genetic variance and/or nondirectional dominance variance. Contrariwise the lack of variation among families in females but their strong response to inbreeding is indicative of little additive or non-directional dominance variance and the presence of 
small amounts of directional dominance. This difference between the sexes is mirrored in the within- and between-population crosses (Table 2). Taken together these results suggest that the genetic architecture of immune response, as measured by encapsulation rate, is strongly affected by the sex in which the genes find themselves (epistasis) and/or there are sex-linked effects. Our studies is consistent with previous studies in Scathophaga stercoraria (Schwarzenbach et al., 2005) and Panorpa vulgaris (Kurtz and Sauer, 1999), who have found only significant sirer component but not dam component in their estimates of heritabilities of immune function. Thus, in the light of these studies, it seems possible that this sex differences in genetic architecture in immune function is widespread among insects.

With respect to the other traits examined (development time, pupal mass, survival), we also found, in general, evidence for genetic variation, though differences between the sexes in response to inbreeding (Table 1, development time, pupal mass) again suggests that the realization of genetic determination differs between the sexes, which could result from epistatic interactions or perhaps sex-linked effects. Interestingly, Saccheri et al. (2005) found in the butterly, Bicyclus anynana, sex differences in the effects of inbreeding on fitness suggesting that trait-specific developmental properties and cryptic selection play crucial roles in shaping genetic architecture.

The present results demonstrate that it cannot be assumed that in invertebrates, unlike vertebrates, there is sexual dimorphism in immunity. Further, these responses may be a function of different genetic architectures and hence evolutionary responses may differ between the sexes. Such potential disparity in response poses interesting questions for the interaction between population dynamics and genetical factors. More detailed research on the components of the genetic variance in male and female moths is needed to elucidate the precise mechanism and to ascertain if such a mechanism is plausibly general or specific to this species.

\section{Acknowledgements}

We thank Janne Kilpimaa, Teija Ruuhola and two anonymous referee, who gave fruitful comments on the manuscript. Special thanks to Ulla Anttila, Tiia Lampela and Liisa M Rantala for assistance in the laboratory. This study was supported by the Academy of Finland to MJR (Projects: 202624 and 212712) and Kone foundation.

\section{References}

Adamo SA, Jensen M, Younger M (2001). Changes in lifetime immunocompetence in male and female Gryllus texensis (formely G. integer): trade-offs between immunity and reproduction. Anim Behav 62: 417-425.

Ahtiainen JJ, Alatalo RV, Kortet R, Rantala MJ (2004). Sexual advertisement and immune function in the wolf spider Hydrolycosa rubrofasciata. Behav Ecol 15: 602-606.

Ahtiainen JJ, Alatalo RV, Kortet R, Rantala MJ (2005). A tradeoff between immune function and sexual signalling in a wild population of the drumming wolf spider Hygrolycosa rubrofasciata. I Evol Biol 18: 985-991.

Calleri II DV, McGrail Reid E, Rosengaus RB, Vargo EL, Traniello JFA (2006). Inbreeding and disease resistance in a social insect: effect of heterozygosity on immunocompetence in the termite Zootermopsis angusticollis. Proc Roy Soc London B 273: 2633-2640.

Fedorka KM, Zuk M, Mousseau TA (2004). Immune suppression and the cost of reproduction in the ground cricket, Allonemobious socius. Evolution 56: 2478-2485.

Gabriel W, Ferriere R (2004). From individual interactions to population viability. In: Ferriere R, Dieckmann U, Couvet D (eds). Evolutionary Conservation Biology. Cambridge University Press: Cambridge. pp 19-40.

Gerloff CU, Ottmer BK, Schmid-Hempel P (2003). Effects of inbreeding on immune response and body size in a social insect, Bombus terrestris. Funct Ecol 17: 582-589.

Gillespie JP, Kanost MR, Trenczek T (1997). Biological mediators of insect immunity. Annu Rev Entomol 42: 611-643.

Gorman MJ, Cornel AJ, Collins FH, Paskewitz SM (1996). A shared genetic mechanism for melanotic encapsulation of CM-sephadex beads and the malaria parasite, Plasmodium cynomolgi B, in the mosquito Anopheles gambiae. Expert Parasitol 84: 380-386.

Gray DA (1998). Sex differences in susceptibility of house crickets, Acheta domesticus, to experimental infection with Serratia liquefaciens. I Invert Pathol 71: 288-289.

Gupta AP (2001). Immunology of invertebrates: cellular. In: Encyclopedia of Life Sciences. Nature Publishing Group: London, UK (www.els.net).

Hanski I (2005). The Shrinking World Ecological Consequences of Habitat Loss. International Ecology Institute: Oldendorf.

Haukioja E (2003). Putting the insect into the birch-insect interaction. Oecologia 136: 161-168.

Haukioja E (2005). Plant defenses and population fluctuations of forest defoliators: mechanism-based scenarios. Ann Zool Fenn 42: 313-325.

Kapari L, Haukioja E, Rantala MJ, Ruuhela T (2006). Immune defence of a defoliating insect interacts with induced plant defence during a population outbreak. Ecology 87: 291-296.

Keller LF, Waller DM (2002). Inbreeding effects in wild populations. Trends Ecol Evol 17: 230-241.

Koskimäki J, Rantala MJ, Suhonen J, Taskinen J, Tynkkynen K (2004). Immunocompetence and resource holding potential in the damselfly Calopteryx virgo L. Behav Ecol 15: 169-173.

Kurtz K, Sauer KP (1999). The immunocompetence handicap hypothesis: testing the genetic predictions. Proc R Soc Lond B 266: 2515-2522.

Kurtz J, Wiesner A, Götz P, Sauer K (2000). Gender differences and individual variation in the immune system of the scorpionfly Panorpa vulgaris (insecta: mecoptera). Dev Comp Immunol 24: 1-12.

Mallon EB, Loosli R, Schmid-Hempel P (2003). Specific versus nonspecific immune defense in the bumblebee, Bombus terrestris L. Evolution 57: 1444-1447.

McKean KA, Nunney L (2005). Batemańs principle and immunity: phenotypically plastic reproductive strategies predict changes in immunological sex differences. Evolution 59: 1510-1517.

Nappi AJ, Vass E, Frey F, Carton Y (1995). Superoxidase anion generation in Drosophila during melanotic encapsulation of parasites. Eur J Cell Biol 68: 450-456.

Paskewitz S, Riehle MA (1994). Response of Plasmodium refractory and susceptible strains of Anopheles gambiae to inoculated sephadex beads. Dev Comp Immunol 18: 369-375.

Rantala MJ, Ahtiainen JJ, Suhonen J (2004). Fluctuating asymmetry and immune function in a field cricket. Oikos 107: 479-484.

Rantala MJ, Jokinen I, Kortet R, Vainikka A, Suhonen J (2002). Do pheromones reveal immunocompetence? Proc Roy Soc London B 269: 1681-1685.

Rantala MJ, Kortet R (2003). Courtship song and immune function in the field cricket Gryllus bimaculatus? Biol J Linn Soc 79: 503-510. 
Rantala MJ, Kortet R, Kotiaho JS, Vainikka A, Suhonen J (2003a). Condition dependence of pheromones and immune function in the grain beetle Tenebrio molitor. Funct Ecol 17: 534-540.

Rantala MJ, Kortet R, Vainikka A (2003b). The role of juvenile hormone in immune function and pheromone production trade-offs: a test of the immunocompetence handicap principle. Proc Roy Soc London B 270: 2257-2261.

Rantala MJ, Koskimäki J, Taskinen J, Tynkkynen K, Suhonen J (2000). Immunocompetence, developmental stability and wing spot size in the damselfly Calopteryx splendens L. Proc Roy Soc London B 267: 2453-2457.

Rantala MJ, Roff DA (2005). An analysis of trade-off in immune function, body size and development time in the Mediterranean field cricket, Gryllus bimaculatus. Funct Ecol 19: 323-330.

Rantala MJ, Roff DA (2006). Analysis of the importance of genotypic variation, metabolic rate, morphology, sex and development time on immune function in the cricket, Gryllus firmus. J Evol Biol 19: 834-843.

Rantala MJ, Roff DA, Rantala LM (2007). Forceps size and immune function in the european earwig Forficula auricularia. Biol J Linn Soc, in press.

Ruohomäki K, Tanhuanpää M, Ayres MP, Kaitaniemi P, Tammatu T, Haukioja E (2000). Causes of cyclicity of Epirria autumnata (Lepidoptera, Geometridae): grandiose theory and tedious practice. Popul Ecol 42: 211-223.

Saccheri IJ, Lloyd HD, Helyar SJ, Brakefield PM (2005) Inbreeding uncovers fundamental differences in the genetic load affecting male and female fertility in a butterfly. Proc Roy Soc B 272: 39-46.

Salt G (1970). The Cellular Defence Reactions of Insects. Cambridge University Press: Cambridge.

Schwarzenbach GA, Hosken DJ, Ward PI (2005). Sex and immunity in the yellow dung fly Scathophaga stercoraria. J Evol Biol 18: 455-463.

Sheridan LAD, Poulin R, Ward DF, Zuk M (2000). Sex differences in parasitic infections among arthropod hosts: is there a male bias? Oikos 88: 327-334.
Simmons LW, Zuk M, Rotenberry JT (2005). Immune function reflected in calling song characteristics in a natural population of the cricket Teleogryllus commodus. Anim Behav 69: 1235-1241.

Stevens L, Yan G, Pray LA (1997). Consequences of inbreeding on invertebrate host susceptibility to parasitic infection. Evolution 51: 2032-2039.

Swindell WR, Bouzat JL (2006). Ancestral inbreeding reduces the magnitude of inbreeding depression in Drosophila melanogaster. Evolution 60: 762-767.

Tammaru T, Ruohomäki K, Saikkonen K (1996). Components of male fitness in relation to body size in Epirrita autumnata (Lepidoptera, Geometridae). Ecol Entomol 21: 185-192.

Teder T, Tammaru T (2005). Sexual size dimorphism within species increases with body size in insects. Oikos 108: 321-334.

Trivers RL (1972). Parental investment and sexual selection. In: Cambell B (ed). Sexual Selection and the Descent of Man, 1871-1971. Heinemann: London. pp 136-179.

Vainio L, Hakkarainen H, Rantala MJ, Sorvari J (2004). Individual variation in immune function in Formica exsecta: effect of nest, body size and sex. Evol Ecol 18: 75-84.

van Ooik T, Rantala MJ, Saloniemi I (2007). Diet-mediated effects of heavy metal pollution on growth and immune response in the geometrid moth, Epirrita autumnata. Environ Pollut 145: 348-354.

Vilmos P, Kurucz E (1998). Insect immunity: evolutionary roots of the mammalian innate immune system. Immunol Lett 62: 59-66.

Yang S, Ruuhola T, Rantala MJ (2007). Impacts of starvation on immune defence and other life history traits of an outbreaking geometrid, Epirrita autumnata: a possible causal trigger of the crash phase of population cycle. Ann Zool Fenn (in press).

Zuk M, Simmons LW, Rotenberry JT, Stoehr AM (2004). Sex differences in immunity in two species of field crickets. Can I Zool 82: 627-634.

Zuk M, Stoehr AM (2002). Immune defence and host life history. Am Nat 160: S9-S22. 